C-A/AP/\#216

September 2005

\title{
Transverse Beam Size Matrices and Beam Emittances for Linearly Coupled Optics
}

\author{
Yun Luo
}

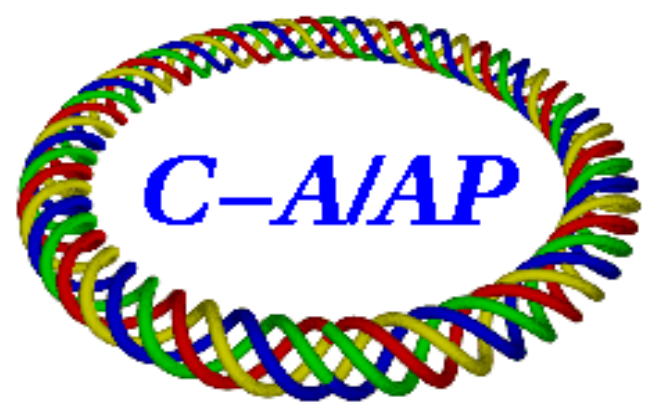

Collider-Accelerator Department Brookhaven National Laboratory Upton, NY 11973 


\title{
Transverse beam size matrices and beam emittances for linearly coupled optics
}

\author{
Yun Luo* \\ Brookhaven National Laboratory, Upton, NY 11973, USA
}

(Dated: August 31, 2005)

\begin{abstract}
Two transverse eigenmode rms emittances are defined statistically in the uncoupled normalized coordinate system. They are assumed to be adiabatic invariants. With linear coupling's action-angle parameterization, the strict expression for the beam size matrix in the laboratory coordinate system is obtained. It can be expressed in matrix $\mathbf{P}$ defined in the action-angle parameterization or in Twiss and coupling parameters defined in Edwards-Teng's parameterization, along with the eigenmode rms emittances. Numerical simulation calculations are carried out to check the analytical expressions and to verify the adiabatic eigenmode rms emittance invariants during the skew quadrupole magnet ramping. With linear coupling's matrix perturbation approach, the strict expressions of the horizontal and vertical beam sizes in the laboratory coordinate system are approximated to give the quasi horizontal and vertical beam emittances under the weak linear difference coupling situation.
\end{abstract}

PACS numbers: 29.20.Dh, 29.27.Bd

*Electronic address: yluo@bnl.gov 


\section{INTRODUCTION}

Under the linearly coupled situation, a single particle's motion is contributed from two eigenmode modes. It doesn't trace out an ellipse in $x-x^{\prime}$ or in $y-y^{\prime}$ phase space. Then, the 1-D emittance does not apply to the coupled situation. However, for weak linear difference couping, with the linear coupling's Hamiltonian perturbation approach [1-4], Guignard retained the 1-D emittance definition and gave the quasi horizontal emittance $\epsilon_{x}$ and vertical emittance $\epsilon_{y}$ as $[3,5]$

$$
\begin{aligned}
& \epsilon_{x}=\epsilon_{x, 0}-\frac{\left|C^{-}\right|^{2} / 2}{\Delta^{2}+\left|C^{-}\right|^{2}}\left(\epsilon_{x, 0}-\epsilon_{y, 0}\right), \\
& \epsilon_{x}=\epsilon_{y, 0}+\frac{\left|C^{-}\right|^{2} / 2}{\Delta^{2}+\left|C^{-}\right|^{2}}\left(\epsilon_{x, 0}-\epsilon_{y, 0}\right),
\end{aligned}
$$

where $\epsilon_{x, 0}$ and $\epsilon_{y, 0}$ are the horizontal and vertical emittances without coupling, respectively. $\Delta$ is the fractional uncoupled tune split. $C^{-}$is the difference coupling coefficient,

$$
C^{-}=\frac{1}{2 \pi} \oint \sqrt{\beta_{x} \beta_{y}} k_{s} e^{i\left(\Phi_{x}-\Phi_{y}-\Delta \frac{2 \pi s}{L}\right)} d l, .
$$

where $\beta_{x}$ and $\beta_{y}$ are the uncoupled betatron amplitude functions, $\Phi_{x}$ and $\Phi_{y}$ are the uncoupled betatron phase advances, $k_{s}$ is the skew quadrupole strength, $L$ is the ring's circumference, and $s$ is the distance between the skew quadruple and the reference point to calculate the coupling coefficient.

In the following, I first define two transverse eigenmode rms emittances in the uncoupled normalized coordinate system. They are assumed to be adiabatic invariants. Then, with the linear coupling's action-angle parameterization [6-10], the strict expression of the beam size matrix in the laboratory coordinate system can be obtained. It can be expressed in matrix $\mathbf{P}$ defined in the action-angle parameterization or in Twiss and coupling parameters defined in Edwards-Teng's parameterization, along with the eigenmode rms emittances. Numerical simulations are carried out with the Relativistic Heavy Ion Collider (RHIC) injection optics to check the analytical expression and to verify the adiabatic eigenmode rms emittance invariants during the skew quadrupole magnet ramping. With the linear coupling's matrix perturbation approach, the strict expressions for the horizontal and vertical beam sizes are approximated to give the quasi horizontal and vertical beam emittances under weak linear difference coupling situation. 


\section{EIGENMODE EMITTANCE}

In this session, I briefly review the definition of the 1-D rms emittance and its statistic calculation. Then, the 2-D eigenmode emittance is defined in the uncoupled normalized coordinate system using the linear coupling's action-angle parameterization.

\section{A. 1-D rms emittance}

Statistically, for example, in the $x-x^{\prime}$ phase space, the uncoupled 1-D rms emittanc is defined as $[11,12]$

$$
\epsilon_{x-x^{\prime}, r m s}=\sqrt{\sigma_{x x} \sigma_{x^{\prime} x^{\prime}}-\sigma_{x x^{\prime}}}
$$

where,

$$
\begin{gathered}
\sigma_{x x}=\sigma_{x}^{2}=\frac{\sum_{i=1}^{N}\left(x_{i}-\bar{x}\right)^{2}}{N}, \\
\sigma_{x^{\prime} x^{\prime}}=\sigma_{x^{\prime}}^{2}=\frac{\sum_{i=1}^{N}\left(x_{i}^{\prime}-\overline{x^{\prime}}\right)^{2}}{N}, \\
\sigma_{x x^{\prime}}=\frac{\sum_{i=1}^{N}\left(x_{i}-\bar{x}\right)\left(x_{i}^{\prime}-\overline{x^{\prime}}\right)}{N} .
\end{gathered}
$$

$N$ is the number of particles in the beam, and $\left(\bar{x}, \overline{x^{\prime}}\right)$ are the coordinates of the distribution barycenter. The rms emittance ellipse is

$$
\sigma_{x^{\prime} x^{\prime}} x^{2}-2 \sigma_{x x^{\prime}} x x^{\prime}+\sigma_{x x} x^{\prime 2}=\epsilon_{x-x^{\prime}, r m s}^{2}
$$

whose area is $\pi \epsilon_{x-x^{\prime}, r m s}$. For Gaussian distributions in the phase space, the rms emittance ellipse contains about $40 \%$ of the total number of the beam particles. According to Liouville's theorem, the rms emittance is constant along the ring.

In the 1-D uncoupled situation, the motion of a single particle traces out an ellipse in the $x-x^{\prime}$ phase space. The ellipse equation is

$$
\gamma_{x} x^{2}+2 \alpha_{x} x x^{\prime}+\beta_{x} x^{\prime 2}=2 J_{x}
$$

where $J_{x}$ represents the particle's action. The area of the ellipse is $2 \pi J_{x}$. If the phase ellipse of one particle happens to coincide with the rms emittance ellipse, comparing Eq. (8) and (9), we get

$$
\begin{gathered}
\epsilon_{x-x^{\prime}, r m s}=2 J_{x, r m s}, \\
\sigma_{x}=\sqrt{\beta_{x} \epsilon_{x-x^{\prime}, r m s}}=\sqrt{2 J_{x, r m s} \beta_{x}}, \\
\sigma_{x}^{\prime}=\sqrt{\gamma_{x} \epsilon_{x-x^{\prime}, r m s}}=\sqrt{2 J_{x, r m s} \gamma_{x}} .
\end{gathered}
$$




\section{B. Action-angle Parametrization}

For 2-D coupled transverse motion, a single particle's motion is contributed from two uncoupled eigenmode modes. It doesn't trace out an ellipse in $x-x^{\prime}$ or $y-y^{\prime}$ phase space. Then, the uncoupled 1-D rms emittance defined above does not apply to the coupled situation.

With the linear coupling's action-angle parameterization, the coupled motion can be decoupled into two uncoupled eigenmode motions. There, a single particle's motion in the laboratory coordinate system is [6]

$$
\mathbf{X}=\left(\begin{array}{c}
x \\
x^{\prime} \\
y \\
y^{\prime}
\end{array}\right)=\mathbf{P}\left(\begin{array}{c}
\sqrt{2 J_{I}} \cos \Phi_{I} \\
-\sqrt{2 J_{I}} \sin \Phi_{I} \\
\sqrt{2 J_{I I}} \cos \Phi_{I I} \\
-\sqrt{2 J_{I I}} \sin \Phi_{I I}
\end{array}\right)
$$

$J_{I, I I}$ and $\Phi_{I, I I}$ are the actions and phases of the two eigenmodes. $J_{I, I I}$ are constant for one particle along the ring. $\mathbf{P}$ can be numerically determined from the eigenvectors of the one-turn $4 \times 4$ linear transfer matrix, or from Twiss and coupling parameters defined in Edwards-Teng's parameterization [6],

$$
\mathbf{P}=\left(\begin{array}{cccc}
r \sqrt{\beta_{I}} & 0 & c_{11} \sqrt{\beta_{I I}}-\frac{c_{12} \alpha_{I I}}{\sqrt{\beta_{I I}}} & \frac{c_{12}}{\sqrt{\beta_{I I}}} \\
-\frac{\alpha_{I} r}{\sqrt{\beta_{I}}} & \frac{r}{\sqrt{\beta_{I}}} & c_{21} \sqrt{\beta_{I I}}-\frac{c_{22} \alpha_{I I}}{\sqrt{\beta_{I I}}} & \frac{c_{22}}{\sqrt{\beta_{I I}}} \\
-\frac{c_{12} \alpha_{I}}{\sqrt{\beta_{I}}}-c_{22} \sqrt{\beta_{I}} & \frac{c_{12}}{\sqrt{\beta_{I}}} & r \sqrt{\beta_{I I}} & 0 \\
\frac{c_{11} \alpha_{I}}{\sqrt{\beta_{I}}}+c_{21} \sqrt{\beta_{I}} & -\frac{c_{11}}{\sqrt{\beta_{I}}} & -\frac{\alpha_{I I} r}{\sqrt{\beta_{I I}}} & \frac{r}{\sqrt{\beta_{I I}}}
\end{array}\right) .
$$

The eigenmode I and eigenmode II motions of a single particle have projections in the $x-x^{\prime}, y-y^{\prime}$, and $x-y$ planes, that normally are ellipses. The ellipse areas of the eigenmode I's projections in the $x-x^{\prime}, y-y^{\prime}$ and $x-y$ planes are [6]

$$
\begin{gathered}
\pi \epsilon_{I, x-x^{\prime}}=2 \pi J_{I} \cdot r^{2}, \\
\pi \epsilon_{I, y-y^{\prime}}=2 \pi J_{I} \cdot\left|1-r^{2}\right|, \\
\pi \epsilon_{I, x-y}=2 \pi J_{I} \cdot r\left|c_{12}\right| .
\end{gathered}
$$

The ellipse areas of the eigenmode II's projections in $y-y^{\prime}, x-x^{\prime}$ and $x-y$ planes are [6]

$$
\pi \epsilon_{I I, y-y^{\prime}}=2 \pi J_{I I} \cdot r^{2}
$$




$$
\begin{gathered}
\pi \epsilon_{I, x-x^{\prime}}=2 \pi J_{I I} \cdot\left|1-r^{2}\right| \\
\pi \epsilon_{I, x-y}=2 \pi J_{I I} \cdot r\left|c_{12}\right| .
\end{gathered}
$$

If $r<1$, for example, in the linear difference coupling regime [13], then

$$
\begin{aligned}
\pi \epsilon_{I, x-x^{\prime}}+\pi \epsilon_{I, y-y^{\prime}} & =2 \pi J_{I}, \\
\pi \epsilon_{I I, y-y^{\prime}}+\pi \epsilon_{I, x-x^{\prime}} & =2 \pi J_{I I} .
\end{aligned}
$$

And if $r>1$, for example, in the linear sum coupling regime [13], then

$$
\begin{gathered}
\pi \epsilon_{I, x-x^{\prime}}-\pi \epsilon_{I, y-y^{\prime}}=2 \pi J_{I}, \\
\pi \epsilon_{I I, y-y^{\prime}}-\pi \epsilon_{I, x-x^{\prime}}=2 \pi J_{I I} .
\end{gathered}
$$

\section{2-D Eigenmode Emittances}

Eq. (13) can be rewritten as

$$
\begin{gathered}
\mathbf{X}=\mathbf{P X}_{n} \\
\mathbf{X}_{n}=\left(\begin{array}{c}
x_{n} \\
x_{n}^{\prime} \\
y_{n} \\
y_{n}^{\prime}
\end{array}\right)=\left(\begin{array}{c}
\sqrt{2 J_{I}} \cos \Phi_{I} \\
-\sqrt{2 J_{I}} \sin \Phi_{I} \\
\sqrt{2 J_{I I}} \cos \Phi_{I I} \\
-\sqrt{2 J_{I I}} \sin \Phi_{I I}
\end{array}\right) .
\end{gathered}
$$

$\mathbf{X}_{n}$ is defined as the uncoupled normalized coordinate. A single particle's motion traces out an circle in the $x_{n}-x_{n}^{\prime}$ or $y_{n}-y_{n}^{\prime}$ phase space. Then, statistically, we can define the two eigenmodes' rms emittances in the $x_{n}-x_{n}^{\prime}$ and $y_{n}-y_{n}^{\prime}$ phase spaces,

$$
\begin{aligned}
& \epsilon_{I, r m s}=\sqrt{\sigma_{x_{n} x_{n}} \sigma_{x_{n}^{\prime} x_{n}^{\prime}}-\sigma_{x_{n} x_{n}^{\prime}}}, \\
& \epsilon_{I I, r m s}=\sqrt{\sigma_{y_{n} y_{n}} \sigma_{y_{n}^{\prime} y_{n}^{\prime}}-\sigma_{y_{n} y_{n}^{\prime}}} .
\end{aligned}
$$

For Gaussian distributions in the $x_{n}-x_{n}^{\prime}$ and $y_{n}-y_{n}^{\prime}$ phase spaces,

$$
\begin{gathered}
\sigma_{x_{n} x_{n}}=\sigma_{x_{n}^{\prime} x_{n}^{\prime}}=\sigma_{x_{n}}^{2}=\sigma_{x_{n}^{\prime}}^{2} \\
\sigma_{y_{n} y_{n}}=\sigma_{y_{n}^{\prime} y_{n}^{\prime}}=\sigma_{y_{n}}^{2}=\sigma_{y_{n}^{\prime}}^{2} \\
\sigma_{x_{n} x_{n}^{\prime}}=\sigma_{y_{n} y_{n}^{\prime}}=0
\end{gathered}
$$


And any correlation coefficient between $x_{n}-x_{n}^{\prime}$ plane and $y_{n}-y_{n}^{\prime}$ plane is zero,

$$
\sigma_{x_{n} y_{n}}=\sigma_{x_{n} y_{n}^{\prime}}=\sigma_{x_{n}^{\prime} y_{n}}=\sigma_{x_{n}^{\prime} y_{n}^{\prime}}=0 .
$$

Then, Eqs. (27) and (28) can be rewritten as

$$
\begin{gathered}
\epsilon_{I, r m s}=\sigma_{x_{n} x_{n}}=\sigma_{x_{n}^{\prime} x_{n}^{\prime}}, \\
\epsilon_{I I, r m s}=\sigma_{y_{n} y_{n}}=\sigma_{y_{n}^{\prime} y_{n}^{\prime}} .
\end{gathered}
$$

Comparing these to the uncoupled 1-D situation, we define

$$
\begin{gathered}
\epsilon_{I, r m s}=2 J_{I, r m s}, \\
\epsilon_{I I, r m s}=2 J_{I I, r m s} .
\end{gathered}
$$

Then, considering Eqs. (29) and (30), for Gaussian distributions in the $x_{n}-x_{n}^{\prime}$ and $y_{n}-y_{n}^{\prime}$ phase spaces, we have

$$
\begin{gathered}
\sigma_{x_{n}}^{2}=\sigma_{x_{n}^{\prime}}^{2}=2 J_{I, r m s}, \\
\sigma_{y_{n}}^{2}=\sigma_{y_{n}^{\prime}}^{2}=2 J_{I I, r m s} .
\end{gathered}
$$

Eigenmode rms emittances $\epsilon_{I, r m s}$ and $\epsilon_{I I, r m s}$ are constant along the ring. For Gaussian distributions in $x_{n}-x_{n}^{\prime}$ and $y_{n}-y_{n}^{\prime}$ phase spaces, the rms emittance ellipses contain about $40 \%$ of the total number of the beam particles. In the following section, numerical simulation will be carried out to verify that the eigenmode rms emittances are adabatic invariants.

\section{CALCULATION OF BEAM SIZE MATRIX}

In this section, the strict expression of the beam size matrix in the laboratory coordinate system is derived, and is compared with the numerical simulations based on the RHIC injection optics.

\section{A. Beam size matrix}

In the the laboratory coordinate system, The beam size matrix $\Sigma$ is defined as $[14,15]$

$$
\Sigma_{\mathbf{X}}=\left(\begin{array}{cccc}
\sigma_{x x} & \sigma_{x x^{\prime}} & \sigma_{x y} & \sigma_{x y^{\prime}} \\
\sigma_{x^{\prime} x} & \sigma_{x^{\prime} x^{\prime}} & \sigma_{x^{\prime} y} & \sigma_{x^{\prime} y^{\prime}} \\
\sigma_{y x} & \sigma_{y x^{\prime}} & \sigma_{y y} & \sigma_{y y^{\prime}} \\
\sigma_{y^{\prime} x} & \sigma_{y^{\prime} x^{\prime}} & \sigma_{y^{\prime} y} & \sigma_{y^{\prime} y^{\prime}}
\end{array}\right) .
$$


This is a symmetric matrix, in which there are 10 independent parameters. We define the coupling angle $\phi$ in the $x-y$ plane, which is the rotation angle of the long axis of the matching particle distribution ellipse with respect to $x$ axis,

$$
\tan 2 \phi=\frac{2 \sigma_{x y}}{\sigma_{x x}-\sigma_{y y}}
$$

In the uncoupled normalized coordinated system, for Gaussian distributions of the beam particles in the $x-x^{\prime}$ and $y-y^{\prime}$ phase spaces, the beam size matrix $\Sigma_{\mathbf{X}_{n}}$ is

$$
\Sigma_{\mathbf{X}_{\mathbf{n}}}=\left(\begin{array}{cccc}
\sigma_{x_{n} x_{n}} & \sigma_{x_{n} x_{n}^{\prime}} & \sigma_{x_{n} y_{n}} & \sigma_{x_{n} y_{n}^{\prime}} \\
\sigma_{x_{n}^{\prime} x_{n}} & \sigma_{x_{n}^{\prime} x_{n}^{\prime}} & \sigma_{x_{n}^{\prime} y_{n}} & \sigma_{x_{n}^{\prime} y_{n}^{\prime}} \\
\sigma_{y_{n} x_{n}} & \sigma_{y_{n} x_{n}^{\prime}} & \sigma_{y_{n} y_{n}} & \sigma_{y_{n} y_{n}^{\prime}} \\
\sigma_{y_{n}^{\prime} x_{n}} & \sigma_{y_{n}^{\prime} x_{n}^{\prime}} & \sigma_{y_{n}^{\prime} y_{n}} & \sigma_{y_{n}^{\prime} y_{n}^{\prime}}
\end{array}\right)
$$

that is,

$$
\Sigma_{\mathbf{X}_{\mathbf{n}}}=\left(\begin{array}{cccc}
2 J_{I, r m s} & 0 & 0 & 0 \\
0 & 2 J_{I, r m s} & 0 & 0 \\
0 & 0 & 2 J_{I I, r m s} & 0 \\
0 & 0 & 0 & 2 J_{I I, r m s}
\end{array}\right)=\left(\begin{array}{cccc}
\epsilon_{I, r m s} & 0 & 0 & 0 \\
0 & \epsilon_{I, r m s} & 0 & 0 \\
0 & 0 & \epsilon_{I I, r m s} & 0 \\
0 & 0 & 0 & \epsilon_{I I, r m s}
\end{array}\right) .
$$

\section{B. In the laboratory coordinate system}

Expanding Eq. (25), one obtains

$$
\left\{\begin{array}{l}
x=p_{11} x_{n}+p_{13} y_{n}+p_{14} y_{n}^{\prime} \\
x^{\prime}=p_{21} x_{n}+p_{22} x_{n}^{\prime}+p_{23} y_{n}+p_{24} y_{n}^{\prime} \\
y=p_{31} x_{n}+p_{32} x_{n}^{\prime}+p_{33} y_{n} \\
y^{\prime}=p_{41} x_{n}+p_{42} x_{n}^{\prime}+p_{43} y_{n}+p_{44} y_{n}^{\prime}
\end{array}\right.
$$

Then, considering Eq. (43) and Eqs. (41) - (42), the rms beam sizes in the laboratory system can be calculated,

$$
\begin{gathered}
\sigma_{x x}=2 p_{11}^{2} J_{I, r m s}+2 p_{13}^{2} J_{I I, r m s}+2 p_{14}^{2} J_{I I, r m s} \\
\sigma_{x x^{\prime}}=2 p_{11} p_{21} J_{I, r m s}+2 p_{13} p_{23} J_{I I, r m s}+2 p_{14} p_{24} J_{I I, r m s} \\
\sigma_{x y}=2 p_{11} p_{31} J_{I, r m s}+2 p_{13} p_{33} J_{I I, r m s} \\
\sigma_{x y^{\prime}}=2 p_{11} p_{41} J_{I, r m s}+2 p_{13} p_{43} J_{I I, r m s}+2 p_{14} p_{44} J_{I I, r m s}
\end{gathered}
$$




$$
\begin{gathered}
\sigma_{x^{\prime} x^{\prime}}=2 p_{21}^{2} J_{I, r m s}+2 p_{22}^{2} J_{I, r m s}+2 p_{23}^{2} J_{I I, r m s}+2 p_{24}^{2} J_{I I, r m s}, \\
\sigma_{x^{\prime} y}=2 p_{21} p_{31} J_{I, r m s}+2 p_{22} p_{32} J_{I, r m s}+2 p_{23} p_{33} J_{I I, r m s} \\
\sigma_{x^{\prime} y^{\prime}}=2 p_{21} p_{41} J_{I, r m s}+2 p_{22} p_{42} J_{I, r m s}+2 p_{23} p_{43} J_{I I, r m s}+2 p_{24} p_{44} J_{I I, r m s} \\
\sigma_{y y}=2 p_{31} J_{I, r m s}+p_{32}^{2} J_{I, r m s}+2 p_{33}^{2} J_{I I, r m s} \\
\sigma_{y y^{\prime}}=2 p_{31} p_{41} J_{I, r m s}+2 p_{32} p_{42} J_{I, r m s}+2 p_{33} p_{43} J_{I I, r m s} \\
\sigma_{y^{\prime} y^{\prime}}=2 p_{41}^{2} J_{I, r m s}+2 p_{42}^{2} J_{I, r m s}+2 p_{43}^{2} J_{I I, r m s}+2 p_{44}^{2} J_{I I, r m s} .
\end{gathered}
$$

Or, expressing this in a compact way,

$$
\Sigma_{\mathbf{X}}=\mathbf{P} \Sigma_{\mathbf{X}_{n}} \mathbf{P}^{T}
$$

When all coupling sources are removed, according to Eq. (54), one can prove

$$
\begin{gathered}
\epsilon_{x, 0}=\frac{\sigma_{x, 0}^{2}}{\beta_{x}}=\epsilon_{I, r m s}, \\
\epsilon_{y, 0}=\frac{\sigma_{y, 0}^{2}}{\beta_{y}}=\epsilon_{I I, r m s},
\end{gathered}
$$

where $\epsilon_{x, 0}$ and $\epsilon_{y, 0}$ are the horizontal and vertical emittances without any coupling, respectively. $\sigma_{x, 0}$ and $\sigma_{y, 0}$ are the horizontal and vertical beam rms sizes without any coupling, respectively.

The eigenmode rms emittances remain constant if the particles achieve the equilibrium distribution. For two observation points, say point 1 and point 2 , in the ring, we have [6]

$$
\mathbf{P}_{2}=\mathbf{T}_{1 \rightarrow 2} \mathbf{P}_{1} \mathbf{R}^{-1}\left(\Delta \Phi_{I}, \Delta \Phi_{I I}\right)
$$

where $\mathbf{T}_{1 \rightarrow 2}$ is the $4 \times 4$ transfer matrix from $p_{1}$ to $p_{2}, \mathbf{R}\left(\Delta \Phi_{I}, \Delta \Phi_{I I}\right)$ is the block-diagonal rotation matrix defined in [6], $\Delta \Phi_{I, I I}$ are the eigenmode phase advances from point 1 to point 2. With Eq. (54), one can prove

$$
\left.\Sigma_{\mathbf{X}}\right|_{2}=\left.\mathbf{T}_{1 \rightarrow 2} \Sigma_{\mathbf{X}}\right|_{1} \mathbf{T}_{1 \rightarrow 2}^{T}
$$

$\left.\Sigma_{\mathbf{X}}\right|_{1,2}$ are the beam size matrices at point 1 and 2 , respectively.

For a transport line, if the beam size matrix Eq. (39) in the laboratory coordinate system can be fully decided, then the eigenmode rms emittances and Twiss, coupling parameters can be decided, according to Eqs. (44)-(53). Then, the beam size matrix and Twiss, coupling parameters can be tracked down along the transport line. 


\section{Numerical simulations}

Numerical simulations of the beam size matrices are based on the Relativistic Heavy Ion Collider (RHIC) Blue ring injection optics. Each RHIC ring has three skew quadrupole families, F1, F2, and F3. Coupling is introduced into the originally uncoupled optics by setting F1 and F3's strengths as $\left(k_{s} d l\right)_{1,3}=0.0004 \mathrm{~m}^{-1}$. I choose the entrance of magnet Q4O6 as the observation point.

First, 10,000 particles are created in the uncoupled normalized coordinate system at the observation point. In this process, I select $\sigma_{x_{n}}^{2}=\sigma_{x_{n}^{\prime}}^{2}=2 \times 10^{-7} \mathrm{~m}^{2}, \sigma_{y_{n}}^{2}=\sigma_{y_{n}^{\prime}}^{2}=1 \times 10^{-7}$ $\mathrm{m}^{2}$, that is, the eigenmode rms emittances $\epsilon_{I, r m s}=2 \times 10^{-7} \mathrm{~m} . \mathrm{rad}, \epsilon_{I I, r m s}=1 \times 10^{-7} \mathrm{~m} . \mathrm{rad}$. With 10,000 particles, the beam size matrix in this system is numerically calculated,

$$
\left.\Sigma_{\mathbf{X}_{n}}\right|_{\text {numerical }}=\left(\begin{array}{cccc}
2.02 \times 10^{-7} & -1.64 \times 10^{-9} & -4.17 \times 10^{-10} & 3.71 \times 10^{-10} \\
-1.64 \times 10^{-9} & 1.98 \times 10^{-7} & -9.45 \times 10^{-10} & 1.08 \times 10^{-9} \\
-4.17 \times 10^{-10} & -9.45 \times 10^{11} & 1.00 \times 10^{-7} & 5.97 \times 10^{-10} \\
3.71 \times 10^{-10} & 1.08 \times 10^{-9} & 5.97 \times 10^{-10} & 9.85 \times 10^{-8}
\end{array}\right) .
$$

The statistic distribution error for 10,000 particles is about $1 \%$.

Then, with Twiss and coupling parameters at the observation point, according to Eqs. (25) and (14), the laboratory coordinates of the 10,000 particles are calculated. The beam size matrix in the laboratory coordinate system then is numerically calculated,

$$
\left.\Sigma_{\mathbf{X}}\right|_{\text {numerical }}=\left(\begin{array}{cccc}
8.48 \times 10^{-6} & 5.16 \times 10^{-7} & 6.10 \times 10^{-7} & -9.81 \times 10^{-8} \\
5.16 \times 10^{-7} & 3.45 \times 10^{-8} & 6.45 \times 10^{-8} & -6.47 \times 10^{-9} \\
6.10 \times 10^{-7} & 6.45 \times 10^{-8} & 2.63 \times 10^{-6} & -1.10 \times 10^{-7} \\
-9.81 \times 10^{-8} & -6.47 \times 10^{-9} & -1.10 \times 10^{-7} & 1.18 \times 10^{-8}
\end{array}\right) .
$$

Analytically, knowing the eignmode rms emittances, according to Eq. (54), the beam size matrix in the laboratory coordinate system can be calculated,

$$
\left.\Sigma_{\mathbf{X}}\right|_{\text {analytical }}=\left(\begin{array}{cccc}
8.42 \times 10^{-6} & 5.13 \times 10^{-7} & 6.07 \times 10^{-7} & -9.50 \times 10^{-8} \\
5.12 \times 10^{-7} & 3.44 \times 10^{-8} & 6.52 \times 10^{-8} & -6.34 \times 10^{-9} \\
6.07 \times 10^{-7} & 6.52 \times 10^{-8} & 2.66 \times 10^{-6} & -1.12 \times 10^{-7} \\
-9.50 \times 10^{-8} & -6.35 \times 10^{-9} & -1.12 \times 10^{-7} & 1.19 \times 10^{-8}
\end{array}\right) .
$$

Comparing Eqs. (60) and (61), the analytical predications of the laboratory beam size matrix agree well with the numerical calculations. 


\section{Adiabatic Eiegnmode Emitance Invariants}

The eigenmode rms emittances are invariants if the particles reach equilibrium distribution. This is not true for injection mismatching and fast magnet ramping. However, if the magnet ramping is slow enough to let the particles catch up with the optics change, we assume the eigenmode rms emittances are adiabatic invariants.

Numerical simulation is used to verify the adiabatic eignmode emittance invariants. For the above coupled RHIC Blue ring injection optics and the same eigenmode rms emittances, I keep the skew quadrupole family F2's strength $\left(k_{s} d l\right)_{2}=0.0004 \mathrm{~m}^{-1}$, while ramping skew quadrupole family F1's strength from $\left(k_{s} d l\right)_{1}=0.0004 \mathrm{~m}^{-1}$ to $-0.0004 \mathrm{~m}^{-1}$ linearly in 1000 turns, or in about 0.1 second. Element-by-element tracking for each particle is performed, based on the RHIC Blue ring injection optics. Fig. 1 shows the particle distributions in the $x_{n}-x_{n}^{\prime}$ phase space before and after the magnet ramping. Fig 2 shows the particle distributions in $x-y$ phase space before and after the magnet ramping.

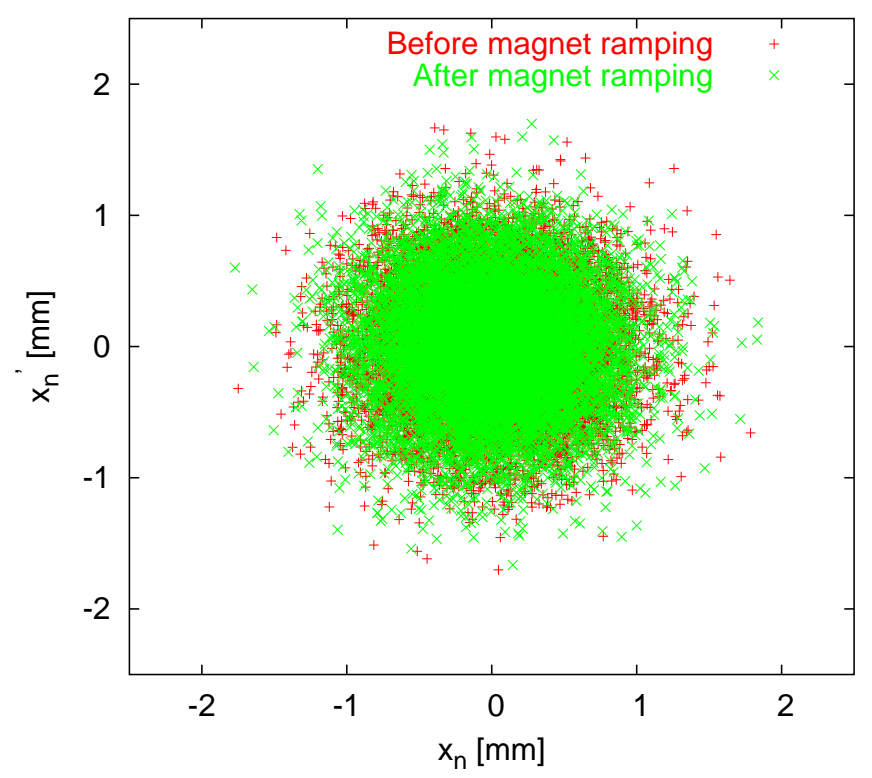

FIG. 1: The particle distributions in $x_{n}-x_{n}^{\prime}$ plane before and after the magnet ramping.

After the magnet ramping, compared to Eq. (60), the beam matrix in the laboratory 


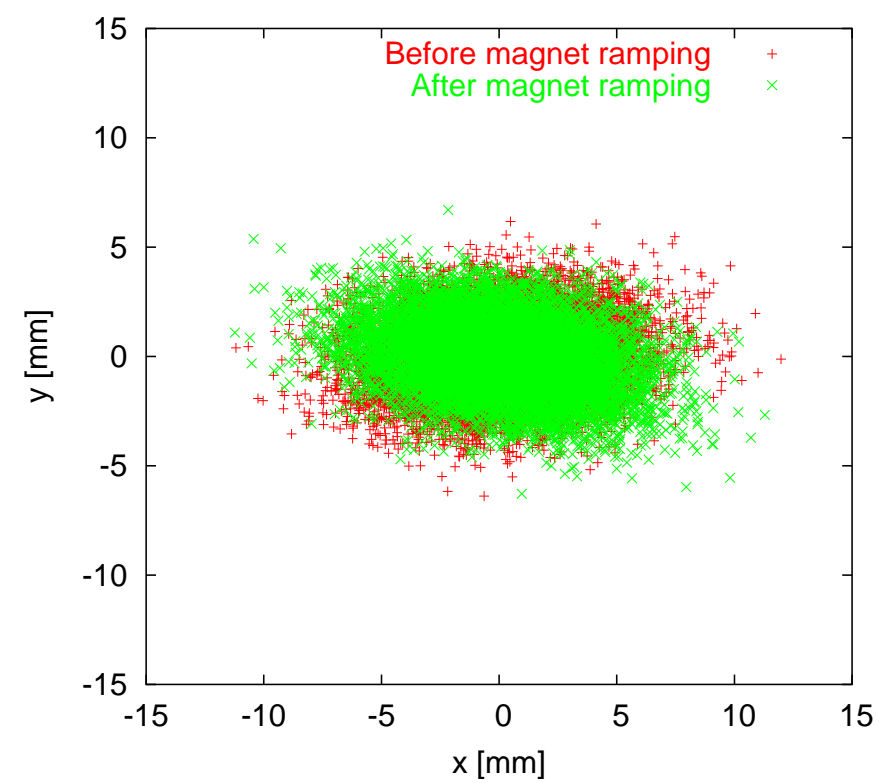

FIG. 2: The particle distributions in $x-y$ plane before and after the magnet ramping..

coordinate system changes to

$$
\left.\Sigma_{\mathbf{X}}\right|_{\text {numerical }}=\left(\begin{array}{cccc}
8.87 \times 10^{-6} & 5.38 \times 10^{-7} & -1.30 \times 10^{-6} & 2.47 \times 10^{-8} \\
5.38 \times 10^{-7} & 3.58 \times 10^{-8} & -6.53 \times 10^{-8} & -4.28 \times 10^{-10} \\
-1.30 \times 10^{-6} & -6.53 \times 10^{-8} & 2.49 \times 10^{-6} & -1.07 \times 10^{-7} \\
2.47 \times 10^{-8} & -4.28 \times 10^{-10} & -1.07 \times 10^{-7} & 1.15 \times 10^{-8}
\end{array}\right) .
$$

$\mathbf{X}_{n}$ is calculated according to Eq. (25) after the magnet ramping. Then, the beam matrix in the uncoupled normalized coordinate system is numerically calculated,

$$
\left.\Sigma_{\mathbf{X}_{n}}\right|_{\text {numerical }}=\left(\begin{array}{cccc}
2.00 \times 10^{-7} & -4.93 \times 10^{-9} & 1.11 \times 10^{-9} & -3.50 \times 10^{-10} \\
-4.93 \times 10^{-9} & 2.01 \times 10^{-7} & 1.74 \times 10^{-10} & -1.66 \times 10^{-9} \\
1.11 \times 10^{-10} & 1.74 \times 10^{11} & 9.87 \times 10^{-8} & 5.87 \times 10^{-10} \\
-3.50 \times 10^{-10} & -1.66 \times 10^{-9} & 5.87 \times 10^{-10} & 1.00 \times 10^{-7}
\end{array}\right) .
$$

Compared to Eq. (59), the eigenmode rms emittances almost have no changes after the magnet ramping.

According to Eq. (40), with the beam size matrices Eqs. (60) and (62) in the numerical simulations, the coupling angle in $x-y$ plane before and after the magnet ramping are calculated,

$$
\begin{gathered}
\left.\phi\right|_{\text {before }, \text { numerical }}=5.89^{\circ}, \\
\left.\phi\right|_{\text {after }, \text { numerical }}=-10.31^{\circ} .
\end{gathered}
$$


Analytically, using $\epsilon_{I, r m s}=2 \times 10^{-7} \mathrm{~m} . \mathrm{rad}$ and $\epsilon_{I I, r m s}=1 \times 10^{-7} \mathrm{~m} . \mathrm{rad}$, and with Twiss and coupling parameters, the coupling angle in $x-y$ plane before and after the magnet ramping are calculated,

$$
\begin{gathered}
\left.\phi\right|_{b e f o r e, \text { analytical }}=5.95^{\circ}, \\
\left.\phi\right|_{\text {before, analytical }}=-10.98^{\circ} .
\end{gathered}
$$

\section{APPROXIMATION TO WEAK LINEAR DIFFERENCE COUPLING}

In this section, the strict expressions of the beam horizontal and vertical sizes are approximated to give the quasi horizontal and vertical beam emittances under weak linear difference coupling situation. The linear coupling's matrix perturbation approach is used.

\section{A. Linear coupling's matrix perturbation approach}

In the linear coupling's matrix perturbation approach, Twiss parameters have no significant changes, $\mathbf{P}$ is then approximately given by $[13,16]$

$$
\mathbf{P}=\mathbf{U} \overline{\mathbf{V}}
$$

where

$$
\begin{gathered}
\mathbf{U}=\left(\begin{array}{cccc}
\sqrt{\beta_{x}} & 0 & 0 & 0 \\
\frac{-\alpha_{x}}{\sqrt{\beta_{x}}} & \frac{1}{\sqrt{\beta_{x}}} & 0 & 0 \\
0 & & \sqrt{\beta_{y}} & 0 \\
0 & 0 & \frac{-\alpha_{y}}{\sqrt{\beta_{y}}} & \frac{1}{\sqrt{\beta_{y}}}
\end{array}\right), \\
\overline{\mathbf{V}}=\left(\begin{array}{cccc}
r & 0 & \bar{c}_{11} & \bar{c}_{12} \\
0 & r & \bar{c}_{21} & \bar{c}_{22} \\
-\bar{c}_{22} & \bar{c}_{12} & r & 0 \\
\bar{c}_{21} & -\bar{c}_{11} & 0 & r
\end{array}\right) .
\end{gathered}
$$

$\overline{\mathbf{C}}$ is the normalized coupling matrix, and $r$ and $\overline{\mathbf{C}}$ are connected by $r^{2}+\|\overline{\mathbf{C}}\|=1$.

Under weak linear difference coupling regime, $r$ and $\overline{\mathbf{C}}$ are approximately [13]

$$
r=\sqrt{\frac{1}{2}+\frac{1}{2} \sqrt{\frac{\sin ^{2} \pi\left(\mu_{x}-\mu_{y}\right)}{\sin ^{2} \pi\left(\mu_{x}-\mu_{y}\right)+\frac{1}{4}\left|h_{-}\right|^{2}}}},
$$




$$
\begin{gathered}
\overline{\mathbf{C}}=\operatorname{sgn}\left(-\frac{\sin \pi\left(\mu_{x}+\mu_{y}\right)}{2 r\left(\cos 2 \pi \mu_{I}-\cos 2 \pi \mu_{I I}\right)}\right) \frac{\sqrt{1-r^{2}}}{\left|h_{-}\right|}\left(\begin{array}{cc}
\operatorname{Re}\left\{h_{-}\right\} & \operatorname{Im}\left\{h_{-}\right\} \\
-\operatorname{Im}\left\{h_{-}\right\} & \operatorname{Re}\left\{h_{-}\right\}
\end{array}\right), \\
h_{-}=\sum_{j=1}^{M}\left(\sqrt{\beta_{x} \beta_{y}} k_{s} d l\right)_{j} e^{i\left[\pi\left(\mu_{x}-\mu_{y}\right)-\left(\phi_{x, j}-\phi_{y, j}\right)\right]}, \\
\operatorname{sgn}(x)=\left\{\begin{array}{c}
1, x>0 \\
0, x<0
\end{array},\right.
\end{gathered}
$$

where $\mu_{x, y}$ are the uncoupled tunes, $\mu_{I, I I}$ are the eigentunes, and $M$ is the number of the skew quadrupoles in the ring.

\section{B. Quasi horizontal and vertical emittances}

Under weak coupling situation, the quasi horizontal and vertical emittances are defined as

$$
\begin{aligned}
\epsilon_{x} & =\frac{\sigma_{x}^{2}}{\beta_{x}}, \\
\epsilon_{y} & =\frac{\sigma_{y}^{2}}{\beta_{y}},
\end{aligned}
$$

where $\sigma_{x}$ and $\sigma_{y}$ are the rms horizontal and vertical beam sizes with coupling, respectively. $\beta_{x}$ and $\beta_{y}$ are the amplitude function without coupling. It should be mentioned that the quasi horizontal and vertical emittances are different from the uncoupled 1-D emittances.

Rewriting Eqs. (44) and (51) as

$$
\begin{aligned}
& \sigma_{x}^{2}=\sigma_{x x}=p_{11}^{2} \epsilon_{I, r m s}+\left(p_{13}^{2}+p_{14}^{2}\right) \epsilon_{I I, r m s}, \\
& \sigma_{y}^{2}=\sigma_{y y}=\left(p_{31}^{2}+p_{32}^{2}\right) \epsilon_{I, r m s}+p_{33}^{2} \epsilon_{I I, r m s},
\end{aligned}
$$

and considering Eqs. (68) - (70),

$$
\begin{aligned}
& \sigma_{x}^{2}=r^{2} \beta_{x} \epsilon_{I, r m s}+\beta_{x}\left(\bar{c}_{11}^{2}+\bar{c}_{12}^{2}\right) \epsilon_{I I, r m s}, \\
& \sigma_{y}^{2}=\beta_{y}\left(\bar{c}_{22}^{2}+\bar{c}_{12}^{2}\right) \epsilon_{I, r m s}+r^{2} \beta_{y} \epsilon_{I I, r m s} .
\end{aligned}
$$

Under weak linear difference coupling situation, according to Eq. (72),

$$
\begin{aligned}
& \sigma_{x}^{2}=r^{2} \beta_{x} \epsilon_{I, r m s}+\beta_{x}\left(1-r^{2}\right) \epsilon_{I I, r m s}, \\
& \sigma_{y}^{2}=\left(1-r^{2}\right) \beta_{y} \epsilon_{I, r m s}+r^{2} \beta_{y} \epsilon_{I I, r m s} .
\end{aligned}
$$


Considering Eqs. (75)- (76) and Eqs. (55)-(56), then Eqs. (81) and (82) can be rewritten as

$$
\begin{aligned}
& \epsilon_{x}=\epsilon_{x, 0}-\left(1-r^{2}\right)\left(\epsilon_{x, 0}-\epsilon_{y, 0}\right), \\
& \epsilon_{y}=\epsilon_{y, 0}+\left(1-r^{2}\right)\left(\epsilon_{x, 0}-\epsilon_{y, 0}\right) .
\end{aligned}
$$

Integrating $r$ from Eq. (71), and considering $\sin \pi\left(\mu_{x}-\mu_{y}\right) \approx \pi \Delta, \Delta$ is the fractional uncoupled tune split, we obtain

$$
\begin{aligned}
& \epsilon_{x}=\epsilon_{x, 0}-\left(\frac{1}{2}-\frac{1}{2} \sqrt{\frac{\Delta^{2}}{\Delta^{2}+\left(\frac{1}{2 \pi} h_{-}\right)^{2}}}\right)\left(\epsilon_{x, 0}-\epsilon_{y, 0}\right), \\
& \epsilon_{y}=\epsilon_{y, 0}+\left(\frac{1}{2}-\frac{1}{2} \sqrt{\frac{\Delta^{2}}{\Delta^{2}+\left(\frac{1}{2 \pi} h_{-}\right)^{2}}}\right)\left(\epsilon_{x, 0}-\epsilon_{y, 0}\right) .
\end{aligned}
$$

Comparing the definitions of $C^{-}$and $h_{-}$, when the accelerator is working close to the linear difference coupling resonance,

$$
\left|C^{-}\right| \approx \frac{1}{2 \pi}\left|h_{-}\right| .
$$

Then, the quasi horizontal and vertical emittances become

$$
\begin{aligned}
& \epsilon_{x}=\epsilon_{x, 0}-\left(\frac{1}{2}-\frac{1}{2} \sqrt{\frac{\Delta^{2}}{\Delta^{2}+\left|C^{-}\right|^{2}}}\right)\left(\epsilon_{x, 0}-\epsilon_{y, 0}\right), \\
& \epsilon_{y}=\epsilon_{y, 0}+\left(\frac{1}{2}-\frac{1}{2} \sqrt{\frac{\Delta^{2}}{\Delta^{2}+\left|C^{-}\right|^{2}}}\right)\left(\epsilon_{x, 0}-\epsilon_{y, 0}\right),
\end{aligned}
$$

and

$$
\epsilon_{x}+\epsilon_{y}=\epsilon_{x, 0}+\epsilon_{y, 0} .
$$

Clearly, Eqs. (88) and Eqs. (89) differ from Eqs. (1) and (2) derived by Guignard using Hamiltonian perturbation approach.

\section{Comparison of different analytical predications}

As an example, I again set the eigenmode rms emittance as $\epsilon_{I, r m s}=2 \times 10^{-7} \mathrm{~m} . \mathrm{rad}$, $\epsilon_{I I, r m s}=1 \times 10^{-7} \mathrm{~m}$. rad for the RHIC Blue injection optics. 10,000 particles with Gaussian distribution are created in the uncoupled normalized coordinate system. The optics is not coupled originally. Then, the skew quadrupole family F1's strength is scanned from 
$\left(k_{s} d l\right)_{1}=-0.0006$ to $0.0006 \mathrm{~m}^{-1}$ with $\Delta\left(k_{s} d l\right)_{1}=0.0001 \mathrm{~m}^{-1}$. Assuming the eigenmode rms emittances are adiabatic invariants, at each scan step, the particles' laboratory coordinates are calculated according to Eq. (25) and $\sigma_{x x}$ and $\sigma_{y y}$ calculated statistically.

During the scan, $\beta_{I}$ is found to have less than $1.6 \%$ deviation from uncoupled $\beta_{x}$, and $\beta_{I I}$ less than $0.3 \%$ deviation from uncoupled $\beta_{y}$. Fig. 3 shows the eigentunes versus the skew quadrupole family F1's strength during the scan. Figs. 4 and 5 show the quasi horizontal emittance $\epsilon_{x}$ and the quasi vertical emittance $\epsilon_{y}$ versus the skew quadrupole family F1's strength, respectively. There, the predications from the strict matrix approach are calculated according to Eq. (54) and Eqs. (75)-(76), while those from the Hamiltonian perturbation approach are calculated according to Eqs.(1) and (2), and those from the matrix perturbation approach are calculated according to Eqs. (88) and (89).

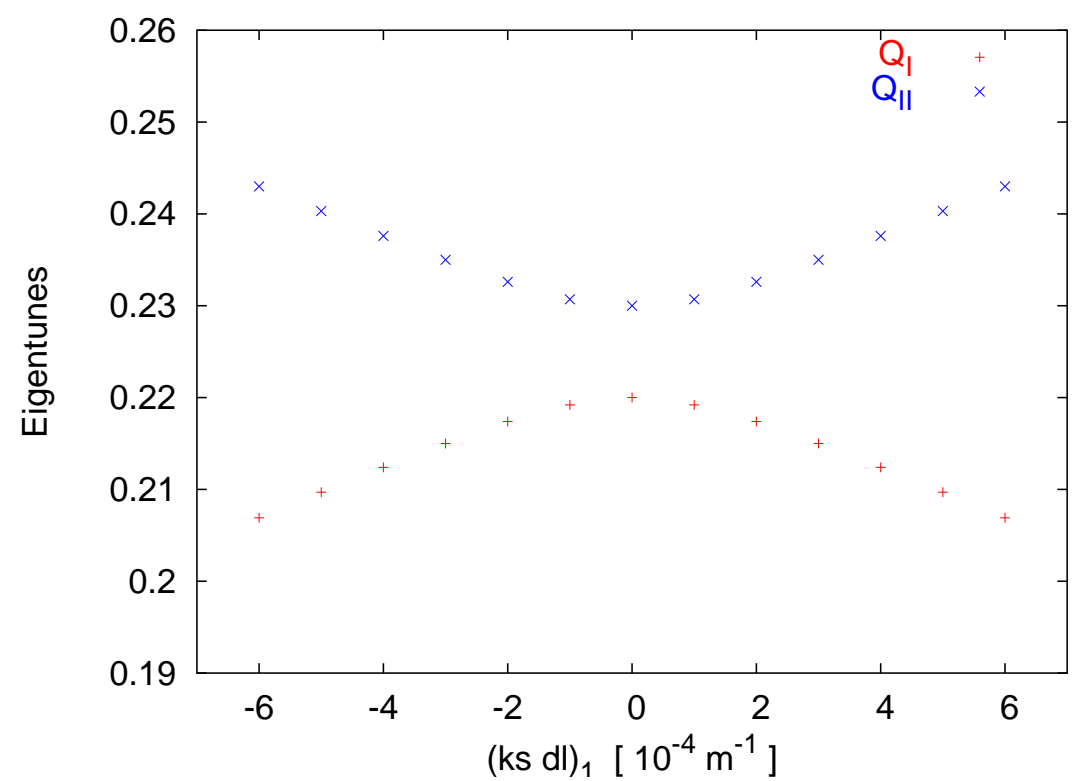

FIG. 3: Eigentunes versus the skew quadrupole family F1's strength in the scan.

Fig.4 and Fig.5 reveal that the strict matrix approach predicts the quasi horizontal and vertical emittances very well. Those from the Hamiltonian perturbation and the matrix perturbation approaches show more or less deviation, especially the former that entails a large predication error in the quasi vertical emittance at $\Delta\left(k_{s} d l\right)_{1}= \pm 0.0006 \mathrm{~m}^{-1}$. 


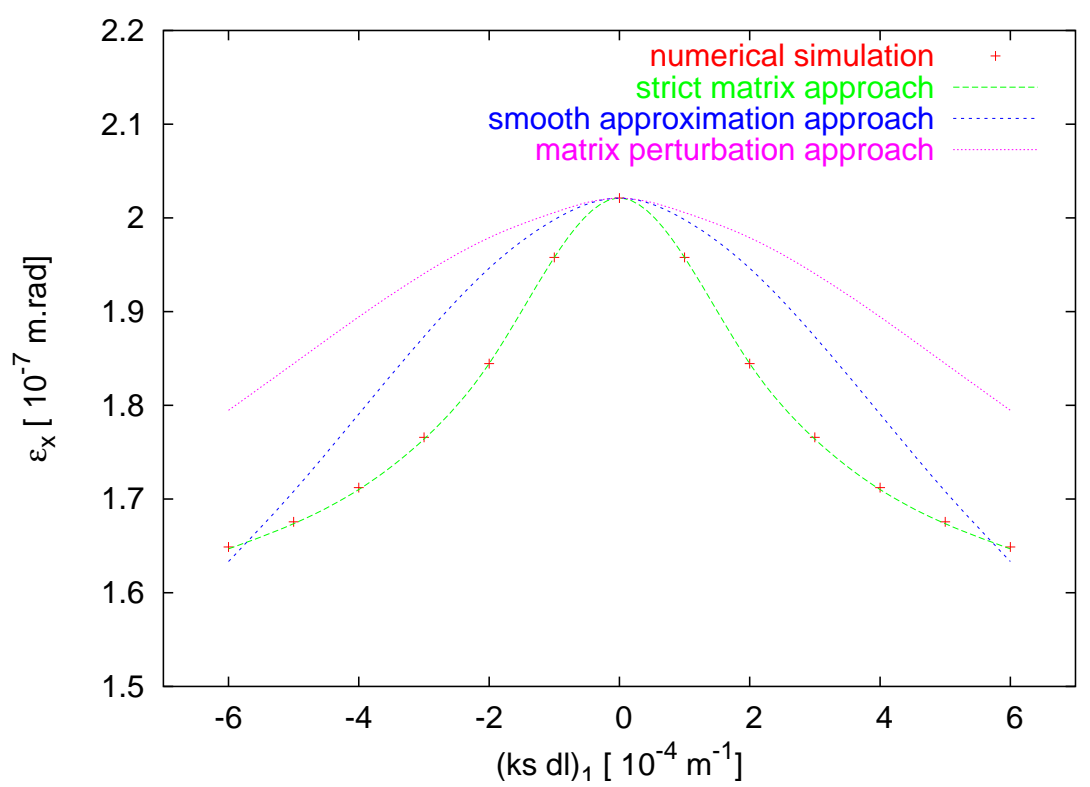

FIG. 4: Quasi horizontal emittance $\epsilon_{x}$ versus the skew quadrupole family F1's strength in the scan.

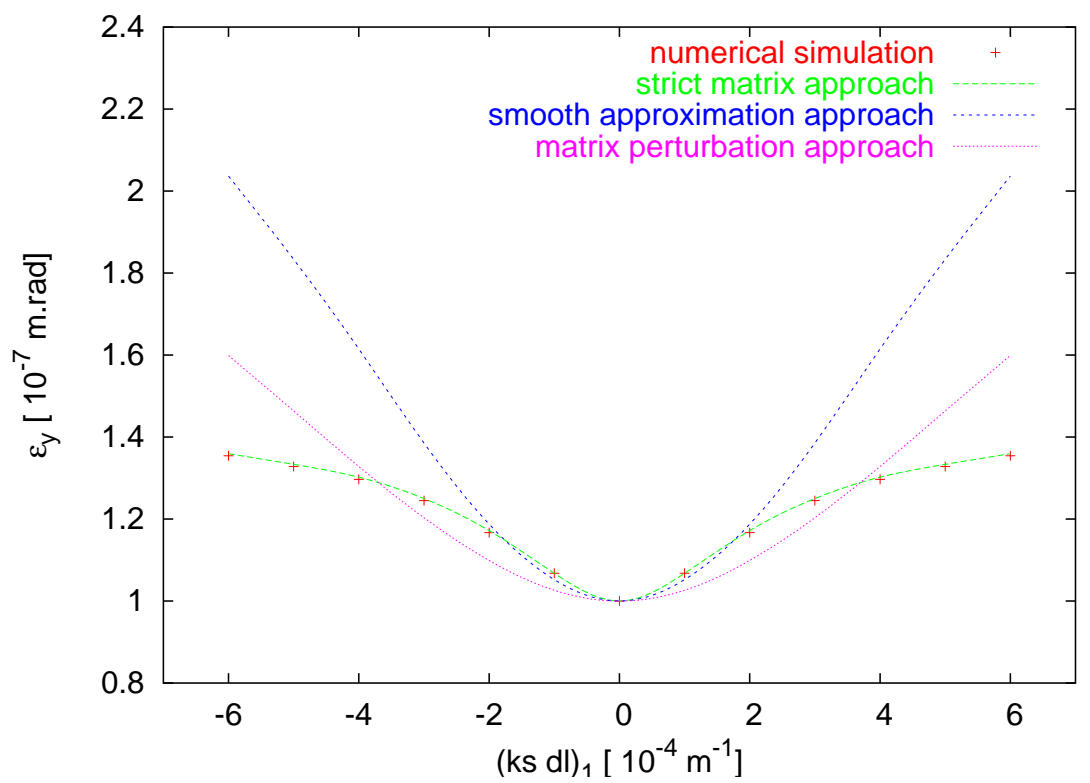

FIG. 5: Quasi vertical emittance $\epsilon_{y}$ versus the skew quadrupole family F1's strength in the scan.

\section{CONCLUSION}

Two transverses eigenmode rms emittances are statistically defined and numerical simulation calculation verifies that they are adiabatic invariants. With the linear coupling's actionangle parameterization, the strict beam size matrix in the laboratory coordinate system is obtained in matrix $\mathbf{P}$ or in Twiss and coupling parameters, together with the eigenmode rms emittances. Under weak linear difference coupling, these strict expressions of the beam sizes 
are approximated to give the quasi horizontal and vertical emittances. Numerical simulation calculations show that the strict matrix approach predicates the beam size matrix and quasi horizontal and vertical emittances very well, while those from the Hamiltonian perturbation and the matrix perturbation approaches have more or less deviation.

\section{ACKNOWLEDGMENTS}

The author thanks A. Chao (SLAC), C. Gardner, V. Lebedev (FNAL), W. W. MacKay, and R. Talman (Cornell) for the discussions of the adiabatic eignmode emittance invariants. And thanks goes to A. Woodhead for her help in preparing this manuscript. This work is supported by U.S. DOE under contract No. DE-AC02-98CH10886.

[1] S. Scoch, CERN Report No. 57-20, 1957 (unpublished).

[2] G. Guignard, CERN Report No. 76-06, 1976 (unpublished).

[3] G. Guignard, Phys. Rev. E 51, p6104, 1995.

[4] H. Wiedemann, Particle Accelerator Physics II, Nonlinear and Higher-Order Beam Dynamics, Springer-Verlag, 1995.

[5] G. Guignard, "Beam Blow-up and Luminosity Reduction due to Linear Coupling", CERN ISR-BOM/77-43, 1977 (unpublished).

[6] Y. Luo, Phys. Rev. ST Accel. Beam 7, 124001 (2004).

[7] D. Sagan and D. Rubin, Phys. Rev. ST Accel. Beam 2, 074001 (1999).

[8] M. Billing, Cornell Report CBN 85-2 ( 1985 ).

[9] S. Peggs, IEEE Trans. Nucl. Sci. 30, 2460 (1983).

[10] D. Edwards and L. Teng, IEEE Trans. Nucl. Sci. 20, 885 (1973).

[11] P.M. Lapostolle, IEEE Trans. Nucl. Sci. NS-18, No 3 , 1101 (1971).

[12] J. Buon, "Beam phase space and emittance", CERN Report No. 94-01, 1994 (unpublished).

[13] Y. Luo, "Matrix perturbation approach to the weak linear coupling",BNL C-AD/AP Note 187, Jan., 2005, (unpublished).

[14] K. Brown, "Transport: a computer program for designing charged-particle beam-transport systems ; 3rd ed", CERN Report No. 80-04, 1980 (unpublished). 
[15] F. C. Iselin, "The MAD Program Version 8.13-Physicist Manual", p.49, unpublished.

[16] Y. Luo, "Simulation Calculations and Analytical Predictions of Eigenmode Projection Parameters",BNL C-AD/AP Note 214, July, 2005, (unpublished). 